\title{
Associations between eating 'style' and dietary behaviour in military personnel: preliminary findings from the UK armed forces
}

\author{
A. Shaw ${ }^{1}$, H. Pearce ${ }^{2}$, T. Davey ${ }^{1}$, J. Harmer ${ }^{3}$, T. Doree ${ }^{4}$, A. Darling ${ }^{2}$, C. Fife-Schaw ${ }^{2}$, \\ S.A. Lanham-New ${ }^{2}$ and J.L. Fallowfield ${ }^{1}$ \\ ${ }^{1}$ Applied Physiology, Institute of Naval Medicine, Alverstoke, Gosport, PO12 2DL, \\ ${ }^{2}$ Nutritional Sciences Department, School of Biosciences and Medicine, University of Surrey, Guildford. GU2 $7 X H$, \\ ${ }^{3}$ Defence Medical Rehabilitation Centre, Headley Court, Epsom, Surrey. KT18 6JW and \\ ${ }^{4}$ Headquarters Home Command, Montgomery House, Aldershot. GU11 2JN, UK.
}

The Armed Forces are not exempt from wider UK societal trends of increasing prevalence of overweight and obesity. Unhealthy body weight adversely impacts on physical and mental health, and in the military is associated with reduced physical fitness, increased risk of injury, and reduced deployability ${ }^{(1-2)}$. Overweight and obesity represent complex and multi-faceted challenges, but maintaining a healthy body weight might be influenced by eating styles (i.e. why people eat and/ or overeat).

The aim of this study was to investigate whether eating style (uncontrolled, restrained, emotional and external) was associated with dietary choice (behaviour) in a sample of military personnel. The identification of dominant eating styles and subsequent food intake is important to identify since it could be used by health professionals to inform dietary therapy, promoting healthy weight maintenance.

The population used in this study was a sample $(n$ 123) of military personnel. Questionnaires detailing demographics and eating style were administered in conjunction with a four-day food diary $(n 101)$, to identify eating style and dietary choice. Seven food groups of interest were identified; fruit and vegetables, sweet foods (e.g. sweet pastries, cakes, biscuits, puddings, custard and confectionary), savoury snacks, energy-dense savoury meals, meat products, sugary soft drinks and alcohol. Data were analysed using SPSS 23 software package; descriptive statistics were determined, and multiple regression analyses were performed where significant correlations were identified.

The sample ( $n 79$ male; $n 44$ female) comprised 42 Officers, 35 Senior Rates and 46 Junior Rates. The mean (SD) age was $36 \cdot 5$ (9.7) years, median (interquartile range, IQR) height was $1.76(0 \cdot 16) \mathrm{m}$, mean body mass was $82 \cdot 1(15 \cdot 5) \mathrm{kg}$, mean waist circumference was $87(12) \mathrm{cm}$ and mean body mass index (BMI) was $26 \cdot 9(3 \cdot 7) \mathrm{kg} \cdot \mathrm{m}^{-2}$.

There were significant associations between: uncontrolled eating and savoury snacks and uncontrolled eating with sweet foods $(\mathrm{P}<0.05 ; r=0.20)$; restrained eating and energy-dense savoury meals $(\mathrm{P}<0.03 ; r=-0.30)$; emotional eating and sweet foods $(\mathrm{P}<0.002 ; r=0.30)$; emotional eating and energy-dense savoury meals $(\mathrm{P}<0.044 ; r=-0.20)$; external eating and sweet foods $(\mathrm{P}<$ 0.002 and $r=0.31)$; and external eating and sugary soft drinks $(\mathrm{P}<0.004 ; r=0.20)$. However, when other variables were controlled (i.e. age, gender, education and rank), food group choice (i.e. eating behaviour) could not be predicted from an individual's preferred eating style. There was a positive correlation between restrained eating and BMI $(\mathrm{P}<0 \cdot 006 ; r=0 \cdot 25)$, where $13.3 \%$ of the variance in BMI could be accounted for by a restrained eating preference.

These preliminary observations provide tentative associations between eating styles and "less healthy" dietary choices. Awareness of these preferences in an individual could inform dietary behaviour change to support healthy weight management. Further research in a larger military cohort is certainly warranted.

(C) Crown Copyright (MOD) 2013: Published with the permission of the Controller of HMSO.

1. Kyrolainen H, Hakkinen K, Kautiainen H et al. (2008) Occupational Medicine 58, 251-256.

2. Blacker SK.Wilkinson DM, Bilzon JL et al. (2008) Military Medicine 173, 278-286. 